\title{
Gastric calcifying fibrous tumor of the stomach presenting as a subepithelial tumor
}

\author{
Bogun Jang ${ }^{1}$, Chang Won $\mathrm{Ha}^{2, \text {, }}$ \\ ${ }^{1}$ Department of Pathology, Jeju National University School of Medicine and Jeju National University Hospital, Jeju, Republic of \\ Korea \\ ${ }^{2}$ Department of Pathology, Cheju Halla General Hospital, Jeju, Republic of Korea
}

\begin{abstract}
We describe a case of a 48-year-old Korean woman who had a subepithelial mass incidentally discovered by endoscopic examination. Endoscopic mucosal resection revealed a well-circumscribed whitish solid mass within the submucosal space. Microscopically, the tumor was comprised of sparse spindle cells in the dense collagenous stroma with several calcifications and lymphoid aggregates. Immunohistochemical analysis showed that the tumor cells are negative for c-kit, smooth muscle actin, desmin, S-100 and CD34. Based on these findings, the tumor was diagnosed with calcifying fibrous tumor.
\end{abstract}

Key words: Calcifying fibrous tumor, Stomach, Subepithelial tumor

\section{INTRODUCTION}

Calcifying fibrous tumor (CFT), also called calcifying fibrous pseudotumor, is a benign mesenchymal tumor characterized by the presence of abundant hyalinized collagen, interspersed bland fibroblastic spindle cells, psammommatous or dystrophic calcifications and lymphoplasmacytic infiltration. ${ }^{1)}$ It usually affects children and young adults and shows a predilection for the subcutaneous and deep soft tissues in the extremities. However, gastric CFT is very rare. Here, we report a case of gastric CFT that has been incidentally detected as a subepithelial mass by esophagogastroduodenoscopy examination.

\section{CASE REPORT}

A 48-year-old female patient was admitted for gastric

Received: April 5, 2019; Revised: April 24, 2019; Accepted: April 25, 2019 Correspondence to : Chang Won Ha

Department of Pathology, Cheju Halla General Hospital, 65 Doryeongro (Yeon-dong), Jeju-city, Jeju Special Self-Governing Province, 63127, Korea

Tel: 82-654-740-5250, FAX: 82-654-743-3110

E-mail: hcw63@hanmail.net tumor that had been incidentally found at medical checkup. The patient underwent an endoscopic evaluation, and which revealed a round subepithelial tumor at greater curvature of high body, and which was an 1-cm sized and relatively well-circumscribed mass. Contrast-enhanced computed tomography scan confirmed a $1 \mathrm{~cm}$ sized, non-enhanced, and homogenous nodular lesion in the same location. Endoscopic mucosal resection was performed.

Cross sections revealed an oval-shaped nodule in the submucosal area, and which showed a whitish hard mass. Grossly, the tumor was covered by intact mucosa. On section, it was a well-defined white mass located in the submucosa, measuring $1 \times 0.6 \mathrm{~cm}$ size. Microscopically, the tumor was characterized by a sparse spindle cell lesion in dense collagenous stroma which was highlighted by Masson's trichrome special stain. Spindle cells had ovoid vesicular nuclei with fine chromatin, but did not show any significant nuclear atypia or pleomorphism. In addition, no mitotic activity was observed. A couple of lymphoid follicles harboring germinal centers or small lymphoid aggregates were scattered in the tumor and a 

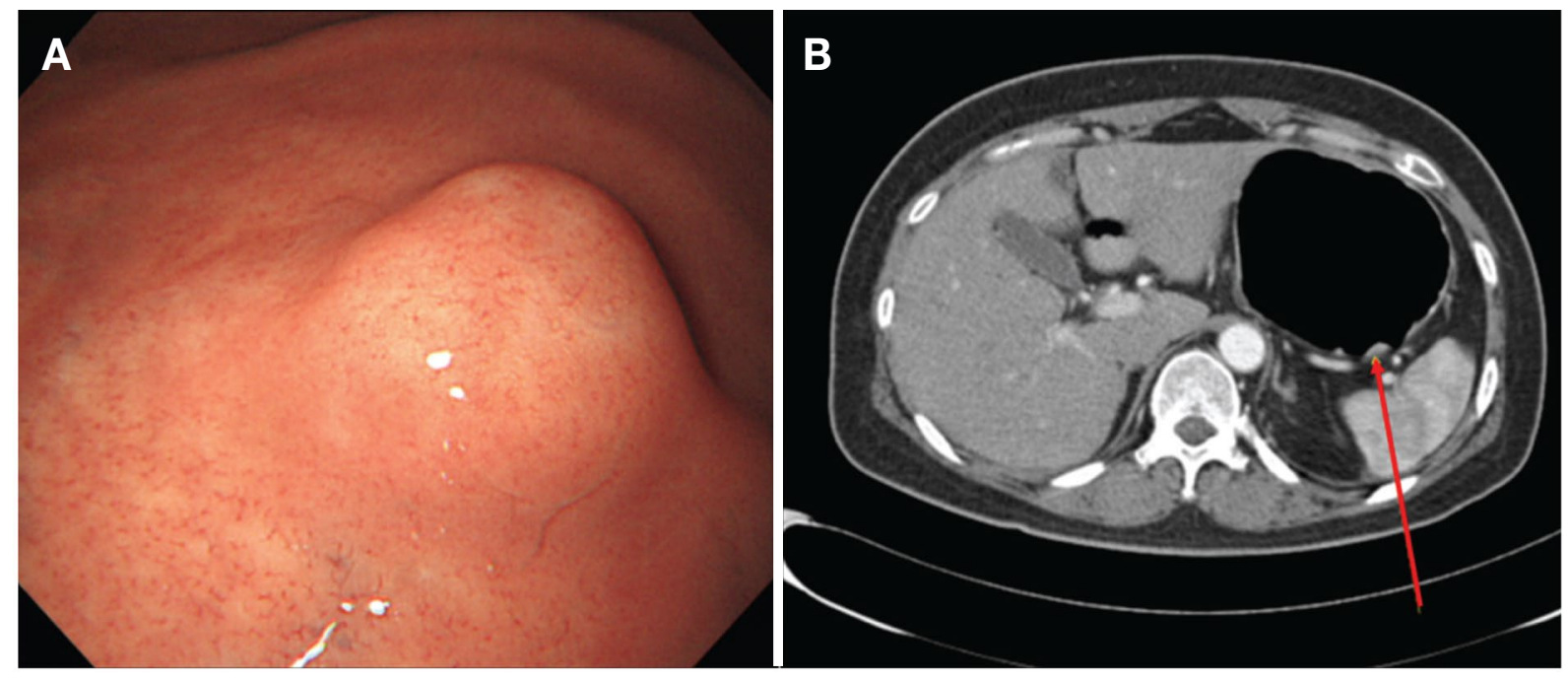

Figure 1. (A) Endoscopic evaluation reveals a polypoid subepithelial mass with intact overlying mucosa at high body of stomach (B) A computed tomography image shows a non-enhanced and homogenous small nodule in the stomach (indicated by red arrow).
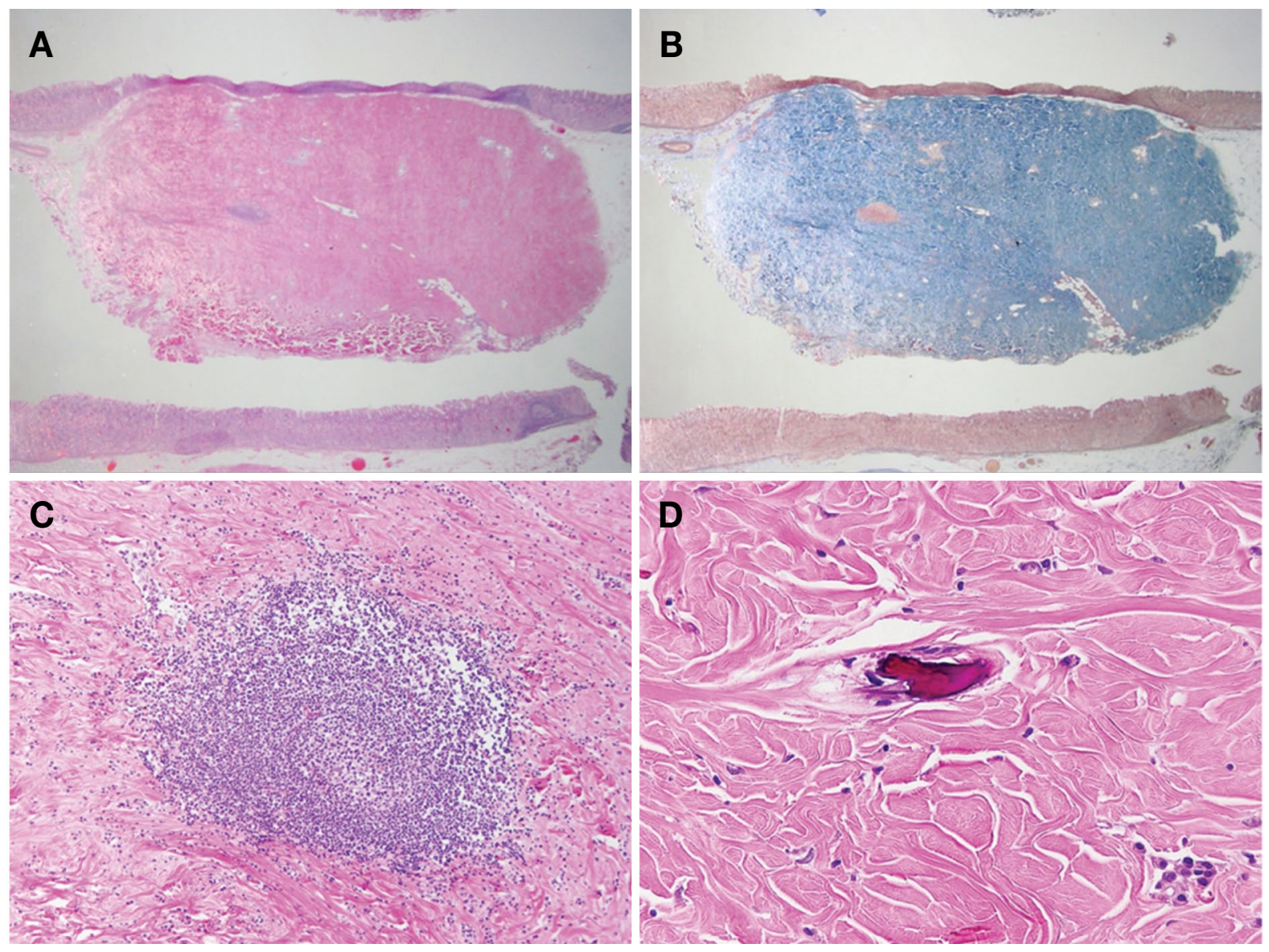

Figure 2. Histologic findings of calcifying fibrous tumor. (A) The tumor is a well-defined oval shaped, with dense collagenous tissue in the submucosa. H\&E $(\times 1.25)$ (B) Special staining highlights the dense fibrotic background. Masson's trichrome $(\times 1.25)(C)$ A lymphoid follicle is observed within the tumor. H\&E $(\times 100)(D)$ Sparse spindle cells are interspersed among thick collagen bundles with dystrophic calcification. H\&E $(\times 400)$ 

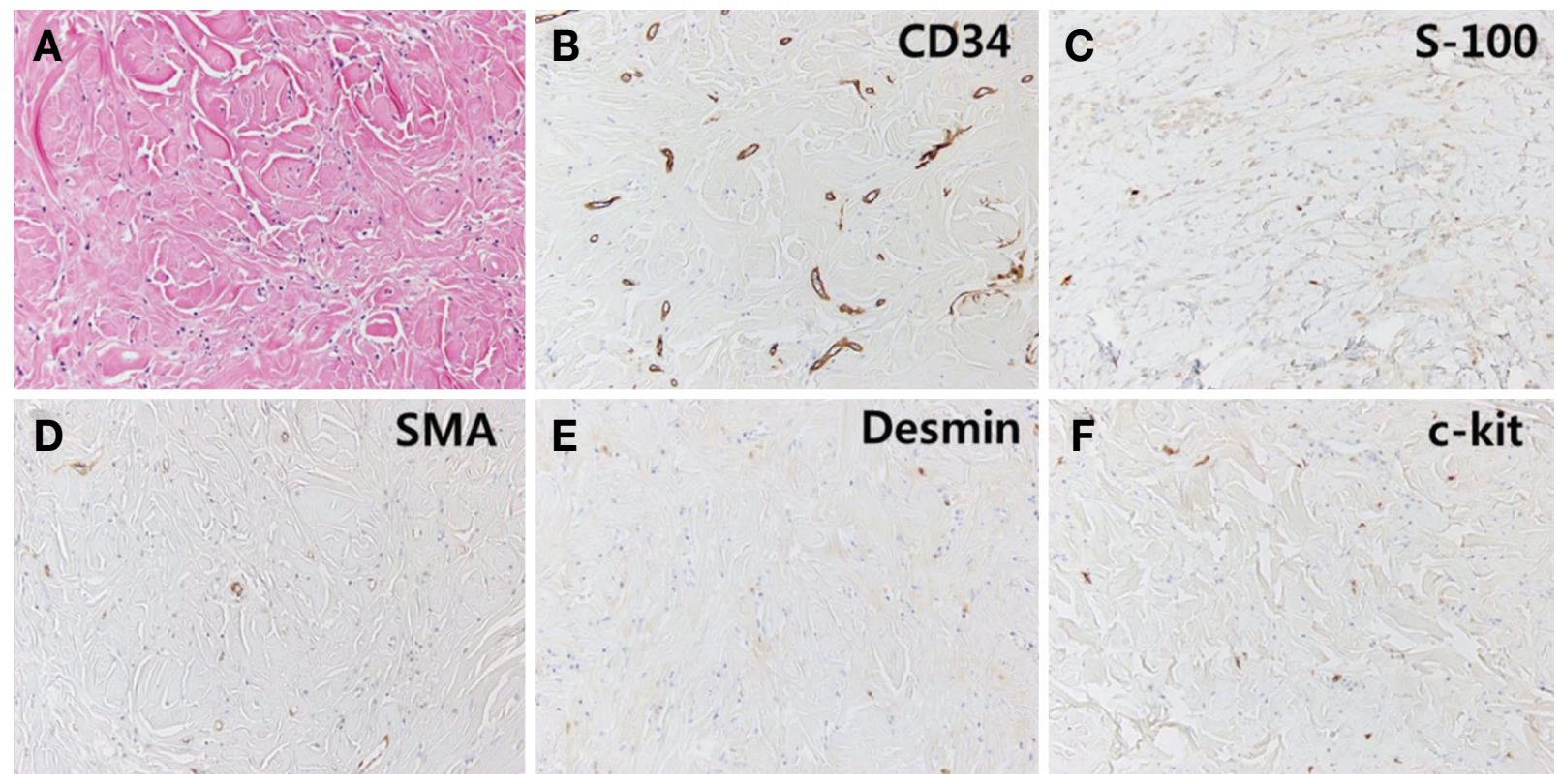

Figure 3. Immunohistochemical analysis for calcifying fibrous tumor. (A) Tumor is characterized by a paucicellular fibroblastic lesion with marked sclerotic stroma. (B-F) Spindle tumor cells showed no immunoreactivity for CD34, S-100, smooth muscle actin, desmin, and c-kit. ( $\times 200)$

couple of dystrophic calcifications were observed. Various inflammatory cells including plasma cells, mast cells, and eosinophils were also found around small vessels. Immunohistochemically, the spindle cells were all negative for CD34, smooth muscle actin (SMA), desmin, S-100, and c-kit.

This study was approved by the Institutional Review Board of Jeju National University Hospital (IRB No. 2018-12-002) and informed consent was waived.

\section{DISCUSSION}

CFT was originally defined as benign, soft, and fibrous mass with psammommatous calcifications in two young girls. ${ }^{2)}$ It is characterized by a heavily collagenized paucicellular spindle cell lesion, psammommatous and/or dystrophic calcifications and varying degrees of inflammatory infiltrates. CFT was initially considered to represent a reactive process, it was also described as calcifying fibrous pseudotumor. ${ }^{1)}$ However, other studies claimed that it is neoplasm with a tendency of non-destructive local recurrence. ${ }^{3)}$

The cause and pathogenesis of CFT remains unclear, but it has been hypothesized that CFT represents a sclerosing end stage of inflammatory fibroblastic tumor (IMT). Despite histologic similarities, including fibroblastic pro- liferation and mixed inflammatory infiltration, CFT has distinctive histologic and immunohistochemical features that are different from IMT. For example, while CFT demonstrates varying degrees of calcifications, IMTs rarely contain calcifications. ${ }^{4)}$ Immunohistochemically, CFTs are diffuse positive for Factor XIIIa and negative for SMA, and CD34, while IMTs are diffusely positive for SMA, variable positivity for CD34 and focal positivity for Factor XIIIa. ${ }^{4)}$

Although gastric CFT is very rare, there have been several reports of CFTs that developed in the stomach..$^{5-13)}$ Gastric subepithelial tumors are mostly asymptomatic and detected incidentally during endoscopic examinations. The most common subepithelial tumors of stomach are gastrointestinal stroma tumors (GISTs) that derived from interstitial cells of Cajal, and other differential diagnosis includes a variety of mesenchymal tumors such as leiomyoma, schwannoma, inflammatory fibroid polyp, inflammatory myofibroblastic tumor, and plexiform fibromyxoma. Histologically, sclerosing GIST, sclerosing leiomyoma and ancient schwannoma may be in part similar to CFT. However, psammommatous calcifications and lymphoplasmastic infiltrates are quite unique features of CFT. In addition, the majority of resected cases are diagnosed as follows based on the immunohistochemical positivity for c-kit (GIST), S-100 (schwannoma), and SMA (leiomyoma). The subepithelial tumor in our patient did 
not express any of these markers and showed typical sclerosing stroma and calcifications, which are consistent with CFT.

Gastric CFT tends to be under-recognized in part due to its low incidence and small size. However, characteristic histology of gastric CFTs may help to recognize them. For example, the calcification detected by endoscopic ultrasonography can be considered a useful feature for suspecting CFTs.

\section{REFERENCES}

1. Fetsch JF, Montgomery EA, Meis JM. Calcifying fibrous pseudotumor. Am J Surg Pathol 1993;17:502-8.

2. Rosenthal N, Abdul-Karim FW. Childhood fibrous tumor with psammoma bodies. Clinicopathologic features in two cases. Arch Pathol Lab Med 1988;112:798-800.

3. Nascimento AF, Ruiz R, Hornick JL, Fletcher CD. Calcifying Fibrous 'Pseudotumor' Clinicopathologic Study of 15 Cases and Analysis of its Relationship to Inflammatory Myofibroblastic Tumor. Int J Surg Pathol 2002;10:189-96.

4. Hill KA, Gonzalez-Crussi F, Chou PM. Calcifying fibrous pseudotumor versus inflammatory myofibroblastic tumor: a histological and immunohistochemical comparison. Mod Pathol 2001; 14:784.

5. Puccio F, Solazzo M, Marciano P, Benzi F. Laparoscopic resection of calcifying fibrous pseudotumor of the gastric wall. A unique case report. Sur Endosc 2001;15:1227.

6. Attila T, Chen D, Gardiner GW, Ptak TW, Marcon NE. Gastric calcifying fibrous tumour. Can J Gastroenterol 2006;20:487-9.

7. Elpek GÖ, Küpesiz GY, Ögüs M. Incidental calcifying fibrous tumor of the stomach presenting as a polyp. Pathol Int 2006;56:22732.

8. Sato S, Ooike N, Yamamoto T, Wada M, Miyamoto A, Matsukawa $\mathrm{M}$, et al. Rare gastric calcifying fibrous pseudotumor removed by endoscopic submucosal dissection. Dig Endosc 2008;20:84-6.

9. Chatelain D, Lauzanne P, Yzet T, Guernou M, Regimbeau JM, Severstre H. Gastric calcifying fibrous pseudotumor, a rare mesenchymal tumor of the stomach. Gastroenterol Clin Biol 2008; 32:441-4.

10. Agaimy A, Bihl MP, Tornillo L, Wünsch PH, Hartmann A, Michal M. Calcifying fibrous tumor of the stomach: clinicopathologic and molecular study of seven cases with literature review and reappraisal of histogenesis. Am J Surg Pathol 2010;34:271-8.

11. Vasilakaki T, Skafida E, Tsavari A, Arkoumani E, Koulia K, Myoteri D, et al. Gastric calcifying fibrous tumor: a very rare case report. Case Rep Oncology 2012;5:455-8.

12. Jang KY, Park HS, Moon WS, Lee H, Kim CY. Calcifying fibrous tumor of the stomach: a case report. J Korean Surg Soc 2012; 83:56-9.

13. Ogasawara N, Izawa S, Mizuno M, Tanabe A, Ozeki T, Noda H, et al. Gastric calcifying fibrous tumor removed by endoscopic submucosal dissection. World J Gastrointest Endosc 2013;5:457. 